\title{
Analysis of the methylation status of genes up-regulated by the demethylating agent, 5-aza-2'-deoxycytidine, in esophageal squamous cell carcinoma
}

\author{
MAKOTO ARAI ${ }^{1,2}$, FUMIO IMAZEKI ${ }^{1}$, YUZO SAKAI ${ }^{2}$, RINTARO MIKATA ${ }^{1}$, MOTOHISA TADA ${ }^{1}$, \\ NAOHIKO SEKI $^{3}$, HIDEAKI SHIMADA ${ }^{4}$, TAKENORI OCHIAI ${ }^{4}$ and OSAMU YOKOSUKA ${ }^{1}$ \\ ${ }^{1}$ Department of Medicine and Clinical Oncology, ${ }^{2} 21$ st Century Center of Excellence \\ (COE) program, Departments of ${ }^{3}$ Functional Genomics, ${ }^{4}$ Academic Surgery, \\ Graduate School of Medicine, Chiba University, Chiba-City, Chiba 260-8670, Japan
}

Received February 1, 2008; Accepted April 2, 2008

DOI: $10.3892 /$ or_00000022

\begin{abstract}
To determine the clinical significance of gene promoter methylation in esophageal squamous cell carcinoma (ESCC), we examined the promoter methylation status of genes showing elevated expression, as determined by DNA microarray-based transcriptomic analysis, in three ESCC cell lines (TE-1, TE-2, TE-10) after 5-aza-2'-deoxycytidine (DAC) treatment. We observed a high degree of DNA methylation within the promoter regions of three genes, namely cathepsin L2 (CTSL2), normal mucosa of esophagus specific 1 (NMES1), and fatty acid binding protein 5 (FABP5). Overexpression of NMES1 in ESCC cell lines increased cell motility. Downregulation of NMES1 might play an important role in the cell motility of ESCC or be a potent marker of malignancy.
\end{abstract}

\section{Introduction}

CG-rich DNA sequences are found within the promoter region and first exon of $\sim 50 \%$ of all genes in the human genome (1). These sequences can be targets of epigenetic phenomena that are associated with altered chromatin structure, transcriptional repression and cancer development

Correspondence to: Dr Makoto Arai, Department of Medicine and Clinical Oncology, Graduate School of Medicine, Chiba University, Inohana 1-8-1, Chiba-City, Chiba 260-8670, Japan E-mail: araim-cib@umin.ac.jp

Abbreviations: ESCC, esophageal squamous cell carcinoma; DAC, 5-aza-2'-deoxycytidine; NMES1, normal mucosa of esophagus specific 1; S.D., standard deviation; HIST1H2BF, histone 1, H2bf; FABP5, fatty acid binding protein 5; CTSL2, cathepsin L2; GADD45A, growth arrest; DNA-damage-inducible, $\alpha$; HIST1H1C, histone $1, \mathrm{H} 1 \mathrm{c}$

Key words: methylation, esophageal squamous cell carcinoma, microarray, normal mucosa of esophagus specific 1
$(2,3)$. With respect to esophageal squamous cell carcinoma (ESCC), hypermethylation events within the promoter regions of p14ARF, p16INK4a, HLA class I genes, E-cadherin, and Ras association (RalGDS/AF-6) domain family 1, have been reported to be related to the development of ESCC (4-7). However, a global analysis of the genes regulated by epigenetic factors in ESCC has rarely been performed.

The nucleotide analogue 5-aza-2'-deoxycytidine (DAC) inhibits DNA methyltransferase, resulting in the reactivation of methylation-silenced genes (8). DAC has been used as an anti-cancer agent for patients with chronic myelogenous leukemia in the expectation of gene reactivation via its demethylation properties (9). In a previous study, we clarified the expression profile of up-regulated genes in hepatoma cell lines by DAC using a DNA microarray-based transcriptomic approach (10).

Herein, we assessed, on a global level, the genes induced by DAC in ESCC cell lines using DNA microarrays, with a goal to find candidate genes regulated by aberrant DNA methylation. From the cohort of genes up-regulated by DAC treatment, we then assessed the methylation status of the promoter $\mathrm{CpG}$ islands of four genes in ESCC cell lines. In addition, we focused on the normal mucosa of esophagus specific 1 (NMES1), which was up-regulated by DAC treatment and displayed abnormal methylation in its promoter region in ESCC cell lines. We report on its function in cancer cell lines.

\section{Materials and methods}

Cell lines. ESCC cell lines (TE-1, TE-2, TE-10) were provided by the Cell Resource Center for Biomedical Research, Tohoku University (11). Cells were maintained in Dulbecco's modified Eagle's medium (DMEM, Sigma-Aldrich Co., St. Louis, MO, USA) supplemented with $10 \%$ fetal bovine serum (FBS; Sanko Junyaku Co., Ltd., Tokyo, Japan). Cell lines were treated with DAC (Sigma-Aldrich Co.), dissolved in DMEM just before use. Cells were grown in medium containing $1 \mu \mathrm{M}$ DAC for $96 \mathrm{~h}$, with the medium and the drug being replaced every $24 \mathrm{~h}$. As a control, cell lines were cultured in normal medium, with the medium also being replaced every $24 \mathrm{~h}$. 
Table I. Primer sets for quantitative RT-PCR analysis.

\begin{tabular}{llccc}
\hline Gene name & Symbol & RefSeq & Sense & Antisense \\
\hline Cathepsin L2 & CTSL2 & NM_001333 & acagtgtggttcttgttggg & ccgttctccttgacatactgg \\
$\begin{array}{l}\text { Normal mucosa of } \\
\text { esophagus specific 1 }\end{array}$ & NMES1 & NM_032413 & aggaactcattcccttggtg & tccacagttcccaaggttc \\
$\begin{array}{l}\text { Growth arrest and } \\
\text { DNA-damage-inducible, } \alpha\end{array}$ & GADD45A & NM_001924 & ggaagtgctcagcaagccc & cgttctcgcagcaaaacgcc \\
Histone 1, H1c & HIST1H1C & NM_005319 & aacagccgtatcaaacttgg & aactggcttcttaggtttgg \\
Actin, 3 & ACTB & NM_001101 & gacaggatgcagaaggagattact & tgatccacatctgatggaaggt \\
\hline
\end{tabular}

Gene name, gene symbol, RefSeq, sense and antisense primers used for PCR amplification and the size of respective PCR products are listed.

DNA and RNA extraction of cell lines. DNA and RNA were extracted from treated and untreated cell lines after the $96 \mathrm{~h}$ time point. Total RNA was prepared using TRIzol reagent (Invitrogen Co., Carlsbad, CA, USA) and DNA was extracted through the use of a QIA amp DNA mini kit (Qiagen Co., Hilden, Germany) according to the manufacturer's instructions.

Microarray analysis. From $5 \mu \mathrm{g}$ of total RNA, RNA probes were generated using a MessageAmp ${ }^{\mathrm{TM}}$ aRNA kit (Ambion Inc., Austin, TX, USA) (http://www.dna-chip.co.jp/thesis/ AceGeneProtocol.pdf). For transcriptomic profiling, we used an oligonucleotide-based DNA microarray, AceGene ${ }^{\circledR}$ (HumanOligoChip30K, DNA Chip Research Inc., Yokohama, Japan). Fluorescence detection was performed essentially as previously described (12). Images were analyzed with Quant Array ${ }^{\circledR}$ (GSI Lumonics, Nepean, Canada) and DNASIS ${ }^{\circledR}$ Array (Hitachi Software Engineering, Tokyo, Japan) according to the manufacturer's instructions. The mean and standard deviation (S.D.) of background levels were calculated and those genes whose intensities were less than the mean plus two S.D. of background levels were excluded from further analysis. The Cy5/Cy3 ratios of all spots on the DNA microarray were normalized by the method of global normalization. Microarray analyses were performed twice with the swapping of $\mathrm{Cy} 3$ or $\mathrm{Cy} 5$ dyes. We evaluated whether there were $\mathrm{CpG}$ islands in the promoter region of genes [from -500 to -1 base pairs (bp)] via CpG Island Searcher (http://ccnt.hsc.usc.edu/ cpgislands2/cpg .aspx) $(13,14)$.

Analysis of mRNA expression by quantitative RT-PCR in cancer cell lines. First-strand synthesis of cDNA was performed with the Superscript First Strand synthesis system for real-time polymerase chain reaction (RT-PCR; Invitrogen) according to the manufacturer's instructions. The cDNA templates were synthesized from $1 \mu \mathrm{g}$ total RNA. Quantitative PCR amplification was performed by Applied Biosystems 7300 (Applied Biosystems, Foster City, CA, USA) according to the manufacturer's instructions using the primer pairs shown in Table I, which shows the predicted sizes of the resulting PCR products. PCR amplification conditions were as follows: pre-incubation $95^{\circ} \mathrm{C}$ for $10 \mathrm{~min}$, with 40 cycles at $95^{\circ} \mathrm{C}$ for $30 \mathrm{sec}$ and $63^{\circ} \mathrm{C}$ for $30 \mathrm{sec}$; $\beta$-actin expression was used as a control (15). TaqMan real-time primers and probes were used for validation when the adequate primers for quantitative RT-PCR could not be designed due to technical difficulties. A set of primers and a probe for each gene tested was obtained from Applied Biosystems. TaqMan ${ }^{\circledR}$ Gene expression assay IDs for the genes were as follows: histone 1, H2bf (HIST1H2BF), Hs00371409_g1; fatty acid binding protein 5 (FABP5), Hs02339439_g1.

Methylation status of the up-regulated genes in cancer cell lines. Using extracted DNA, bisulfite modification of DNA $(1 \mu \mathrm{g})$ was performed through the use of a $\mathrm{Cp}$ Genome ${ }^{\mathrm{TM}}$ DNA modification kit (Chemicon International Inc., Temecula, CA, USA) according to the manufacturer's instructions. To clarify the methylation status of promoter regions, bisulfite genomic sequencing analysis was used. We amplified the bisulfite-modified gene promoters from genomic DNA $(0.04 \mu \mathrm{g})$ via PCR (details of primer sets are given in Table II). PCR conditions were $95^{\circ} \mathrm{C}$ for $15 \mathrm{~min}$ and 40 cycles at $95^{\circ} \mathrm{C}$ for $30 \mathrm{sec}$, annealing temperature for $30 \mathrm{sec}$, and $72^{\circ} \mathrm{C}$ for $90 \mathrm{sec}$, ending with an extension at $72^{\circ} \mathrm{C}$ for $15 \mathrm{~min}$. The amplified DNAs were subcloned into the TA-cloning vector (Invitrogen). We determined the sequence of the promoter in eight independent colonies in each case and examined the extent of methylation by determining the number of methylated cytosine residues.

Selection of NMES1 stable-transfected cells. TE-1 and TE-2 cells were plated in 100-mm culture dishes, incubated overnight, and then transfected with $2 \mu \mathrm{g}$ of plasmid DNA (OmicsLink $^{\mathrm{TM}}$ Expression clone purchased from GeneCopoeia, Inc., Germantown, MD, USA) using lipofectin reagent (Invitrogen), according to the manufacturer's protocol. After incubation in the growth medium for 2 days, cells were fed with DMEM/10\% FBS containing G418 (0.6 mg/ml). After two weeks, the G418-resistant clones were individually picked and expanded. The expression of NMES1 in each individual clone was checked by the RT-PCR method, and their cell viability was examined by the 3-(4,5-dimethylthiazol-2-yl)-5 -(3-carboxymethoxy-phenly)-2-(4-sulfonyl)-2H-tetrazolium 
Table II. Primer sets of PCR amplification of promoter regions of candidate methylated genes.

\begin{tabular}{|c|c|c|c|c|c|}
\hline Gene symbol & Sense & Antisense & Position & Size & ${ }^{\circ} \mathrm{C}$ \\
\hline CTSL2 & aatgtaatagattgggaagg & acaccctcaacaaacaaacc & From -523 to +163 & 686 & 60 \\
\hline NMES1 & gttggaatatagtggtgtaa & cccctacaaaacattctac & From -562 to -101 & 462 & 50 \\
\hline FABP5 & gggttaggtttttaggtgat & ttccaactactaaactataa & From -313 to +80 & 393 & 51 \\
\hline GADD45A (part 1) & gggatttttatatgtggttattagtttttt & acaaattacattaaaaaaatatctccaaacc & From -768 to -449 & 320 & 54 \\
\hline GADD45A (part 2) & ggtagtttgttttagtttaagttgagg & cctaccaaccactaaaaaacaaaaaac & From -323 to +14 & 337 & 51 \\
\hline
\end{tabular}

Indicated gene promoter elements were PCR amplified from bisulfite-modified genomic DNA. Sense and antisense primers used for PCR amplification and the size of respective PCR products are shown. Positions were the distance from the transcription site defined by using the Entrez Gene home page at (www.ncbi.nlm.nih.gov/sites/entrez?db=gene). The primer sets for GADD45A were designed by Wang et al (27).

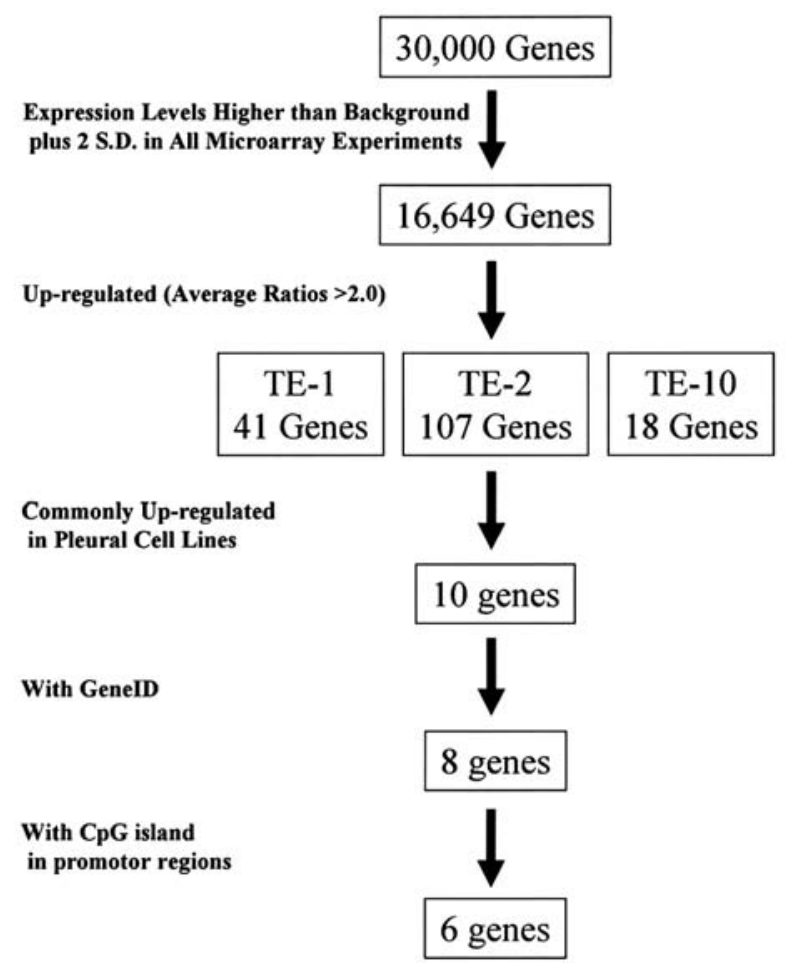

Figure 1. Flowchart for selection of candidate genes regulated by epigenetic factors in esophageal squamous cell carcinoma (ESCC) cell lines. We used three ESCC cell lines to find candidate genes regulated by epigenetic factors. We obtained 16,649 genes that demonstrated expression levels greater than the mean plus two S.D. of background levels in all experiments. Among them, we selected the genes up-regulated (average of ratios $>2.0$ ) by DAC treatment. The numbers of the genes in TE-1, TE-2, and TE-10 cells were 41,107 , and 18 , respectively. We excluded the genes without GeneID or $\mathrm{CpG}$ islands in their promoter regions. As a result, we selected six genes for the examination of the restoration of their expression levels after DAC treatment.

(MTS) assay (CellTiter 96 AQueous One Solution cell proliferation assay; Promega Corp., Madison, WI, USA). The results from six assays per sample were quantified by reading the absorbance at $490 \mathrm{~nm}$ with a microplate colorimeter.

Analysis of the role of NMES1 in cell motility. Cell motility was assessed using the Boyden chamber assay according to the manufacturer's protocol (BD, Franklin Lakes, NJ, USA). To exclude clonal variation, the cell motility was confirmed using two different NMES1 overexpressed clones. Briefly, 200,000 parental (TE-1) and NMES1 overexpressing cell lines (TE1-1 and TE1-4) in optiMEM (Invitrogen) without FBS were each placed on an insert with $8.0-\mu \mathrm{m}$ pore size membrane in 24-well plate, respectively. Six hundred thousand parental (TE-2) and NMES1 overexpressing cells (TE2-3 and TE2-5) were placed into 24-well plates using the same procedure. DMEM plus $10 \%$ FBS was placed in the bottom wells as a chemoattractant. After $24 \mathrm{~h}$, the cells that did not migrate were removed from the top side of the inserts with a cotton swab. Cells that had migrated to the underside of the inserts were stained with Diff-Quik (Sysmex International Reagents Co., Kobe, Japan). After staining, the number of cells on each insert in a field of view was counted at $\times 100$ magnification; this count was performed 5 times. Results were expressed as mean \pm S.D.

Statistical analysis. To determine the statistical significance of the data relating to measurement of cell viability and induction of apoptosis, the Mann-Whitney U test was performed using StatView (SAS Institute Inc., Cary, NC, USA). $p<0.05$ was considered significant.

\section{Results}

Genes up-regulated by DAC treatment revealed by DNA microarray analysis. An oligonucleotide microarray representing 30,000 genes was used. Microarray analyses were performed twice in each cell line with the swapping of $\mathrm{Cy} 3$ or Cy5 dyes. In total, six analyses were performed. The number of genes with expression levels greater than the mean plus two S.D. of background levels in all experiments was 16,649 (Fig. 1); these genes were analyzed further. The number of genes that were up-regulated (average of ratios $>2.0$ ) by DAC treatment in TE-1, TE-2, and TE-10 cells were 41, 107, and 18, respectively. Among them, ten genes were selected due to up-regulation in plural cell lines. Two genes without GeneID, and other two genes without a $\mathrm{CpG}$ island in their promoter regions, were excluded from further analysis. As a result, we studied the expression levels of six genes as follows using the quantitative RT-PCR method: cathepsin L2 (CTSL2), NMES1, FABP5, growth arrest and DNA-damage-inducible, $\alpha$ 

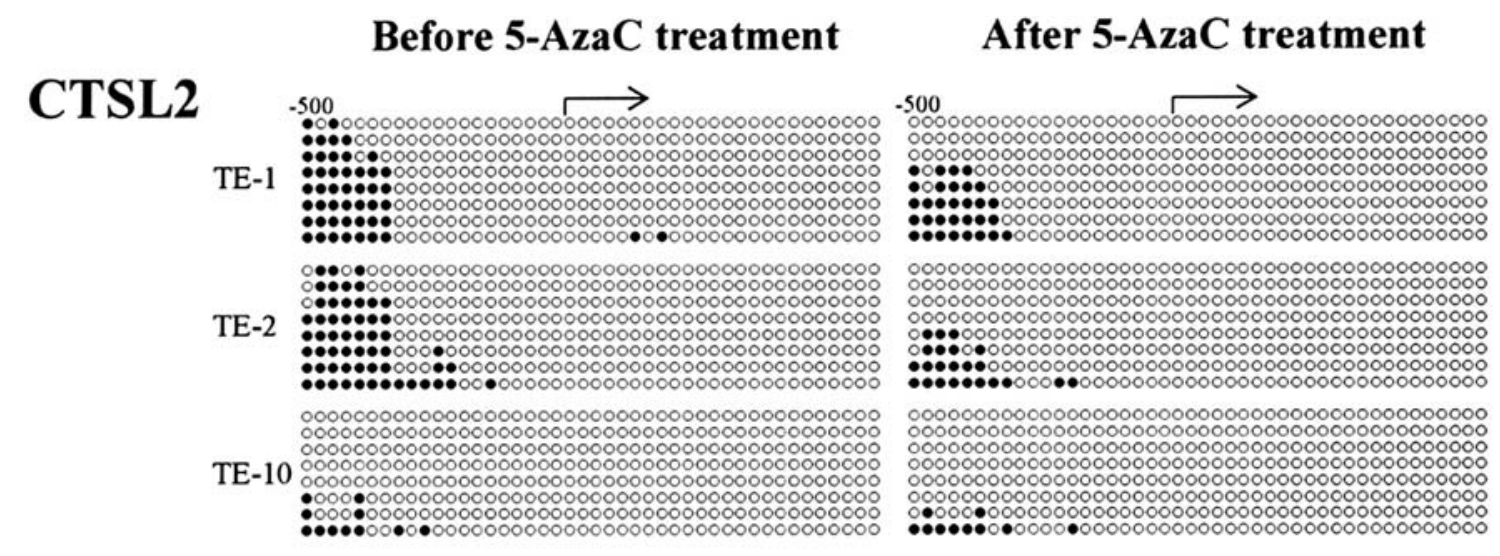

NMES1

TE-1

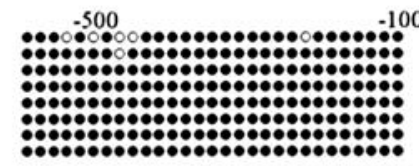

100

0000000000000000000000000000

TE-2

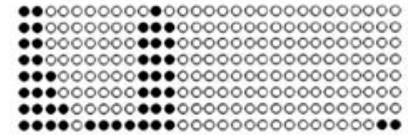

TE-10
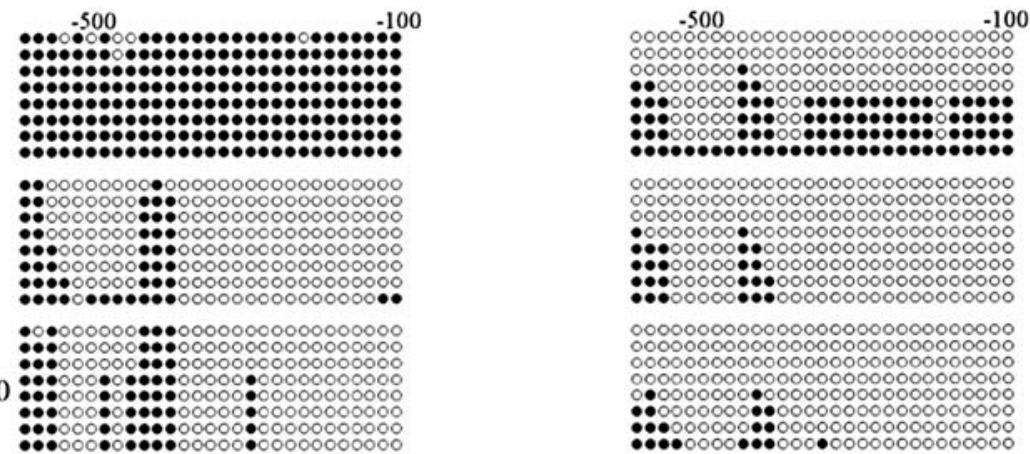

FABP5

TE-1
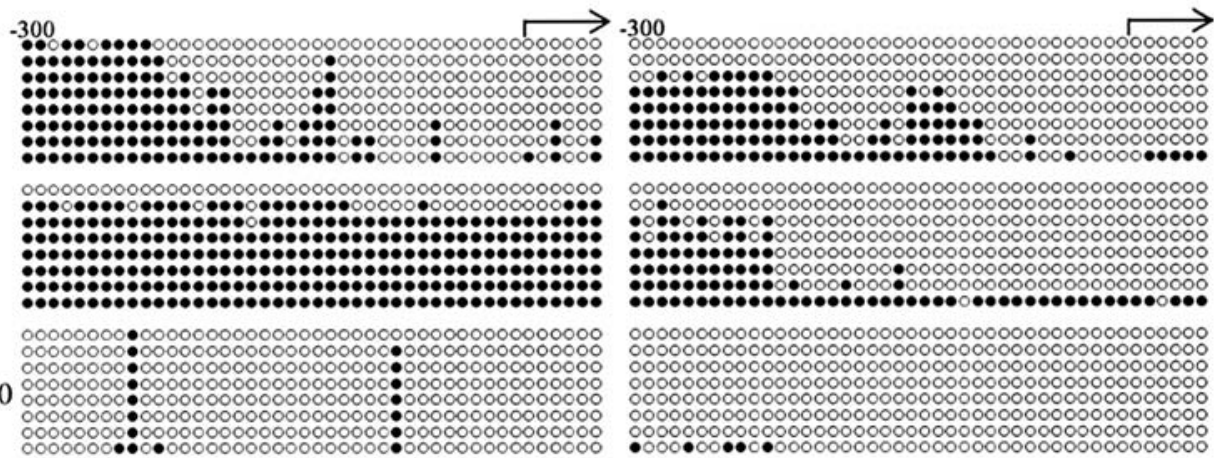

\section{GADD 45A}
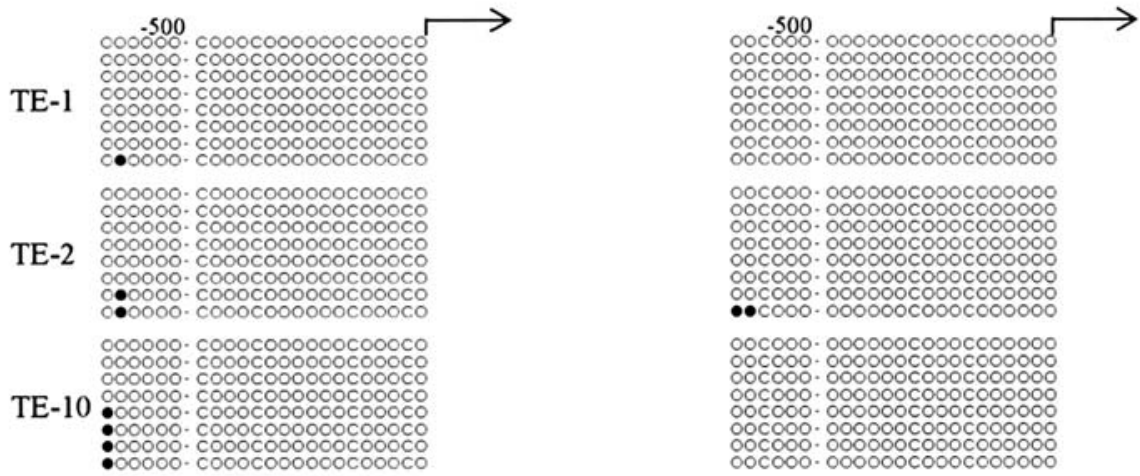

Figure 2. The methylation status of promoter regions of CTSL2, NMES1, GADD45A, and FABP5 in esophageal squamous cell carcinoma (ESCC) cell lines before and after DAC treatment. The methylation status of $\mathrm{CpG}$ islands in each promoter region and first exon were analyzed by a bisulfite genomic sequencing method. After DAC treatment, demethylation effects were obvious in all genes and cell lines. Blank circle, non-methylated; solid circle, methylated. Transcription start sites are as indicated above each methylation mapping. CTSL2, cathepsin L2; NMES1, normal mucosa of esophagus specific 1; FABP5, fatty acid binding protein 5; GADD45A, growth arrest and DNA-damage-inducible, $\alpha$.

(GADD45A), Histone 1, H1c (HIST1H1C), and HIST1H2BF. The results of the microarray and quantitative RT-PCR analyses are shown for the six genes in Table III; restoration of the expression level after DAC treatment was confirmed for four of the six as follows: CTSL2, NMES1, FABP5, and GADD45A. 
Table III. DNA microarray and quantitative RT-PCR data for the genes up-regulated by DAC treatment.

\begin{tabular}{|c|c|c|c|c|c|c|c|}
\hline \multirow[t]{2}{*}{ Gene symbol } & \multirow[t]{2}{*}{ GeneID } & \multicolumn{3}{|c|}{$\begin{array}{l}\text { Ratios of DAC treatment/control } \\
\text { by quantitative RT-PCR }\end{array}$} & \multicolumn{3}{|c|}{$\begin{array}{c}\text { Ratios of DAC treatment/control } \\
\text { by microarray analysis }\end{array}$} \\
\hline & & TE-1 & TE-2 & TE-10 & TE-1 & TE-2 & TE-10 \\
\hline CTSL2 & 1515 & 5.79 & 2.33 & 2.15 & 3.99 & 1.55 & 2.10 \\
\hline NMES1 & 84419 & 4.62 & 8.24 & 1.65 & 2.25 & 3.67 & 1.33 \\
\hline FABP5 & 2171 & 2.47 & 5.47 & 1.01 & 2.30 & 2.89 & 1.60 \\
\hline GADD45A & 1647 & 1.58 & 4.04 & 2.38 & 1.78 & 2.67 & 3.56 \\
\hline HIST1H1C & 3006 & 0.81 & 0.36 & 0.45 & 2.23 & 3.13 & 1.48 \\
\hline HIST1H2BF & 8343 & 40.36 & 0.28 & 0.55 & 52.11 & 2.01 & 1.71 \\
\hline
\end{tabular}
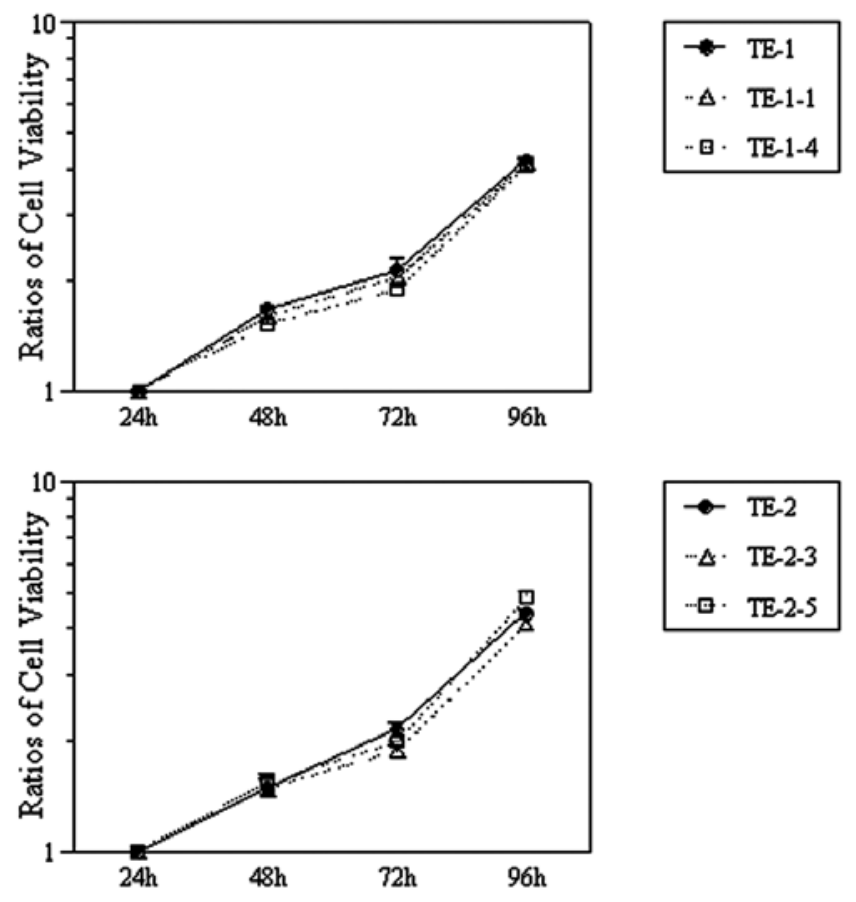

Figure 3. Cell viability in ESCC lines with overexpression of NMES1. The numbers of viable cells were measured by a 3-(4,5-dimethylthiazol-2-yl)-5(3-carboxymethoxy-phenly)-2-(4-sulfonyl)-2H-tetrazolium assay at 24, 48, 72 , and $96 \mathrm{~h}$ after incubation and the ratio at 48, 72, and 96 compared to $24 \mathrm{~h}$ after incubation were calculated. Results are the mean of six independent experiments. There was no significant difference between the cell lines with regard to the overexpression of NMES1 and their parental cells (Mann-Whitney U test). Error bars indicate S.D.

Methylation status of the up-regulated genes in ESCC cell lines. We determined the methylation status of the $\mathrm{CpG}$ islands in the promoter regions of CTSL2, NMES1, FABP5, and $G A D D 45 A$ (Fig. 2). Using bisulfite genomic sequencing analysis, aberrant methylation was observed in the promoter regions of CTSL2, NMES1, and FABP5, with only slight methylation observed in the GADD45A promoter.

Cell motility in ESCC lines with overexpression of NMES1. After selection for 2 weeks, G418-resistant colonies began to appear. Among them, we selected clones which expressed NMES1 stably, and had the same cell viability as the parental cell lines. These were: two sublines (clone TE1-1 and TE1-4) from TE-1 and two (clone TE2-3 and TE2-5) from TE-2 (Fig. 3). Overexpression of NMES1 was confirmed by the quantitative RT-PCR method. The ratios of expression levels of NMES1 in TE1-1 and TE1-4 compared to TE-1 and in TE2-3 and TE2-5 compared to TE-2 were 2.1 $\pm 0.1,1.6 \pm 0.1$, $3.6 \pm 0.4$, and $2.3 \pm 0.2$, respectively. Boyden chamber assays were performed to compare the migratory capability of NMES1 overexpressed cells with the parental ones (Fig. 4A). At $24 \mathrm{~h}$, the number of cells migrating were 111.3 \pm 5.5 , $20.3 \pm 1.5$, and $39.0 \pm 6.2$ for TE-1 (parental cells), TE1-1, and TE1-4 (NMES1 overexpressed cells), respectively. The corresponding numbers for TE-2 (parental cells), TE2-3, and TE2-5 (NMES1 overexpressed cells) were 137.0 \pm 13.7 , $29.0 \pm 4.0$, and 54.7 \pm 22.7 , respectively. There was a significant difference between NMES1 overexpressed cells and parental cells $(\mathrm{p}<0.01)$ (Fig. 4B).

\section{Discussion}

Finding genes that are down-regulated in cancer tissue due to epigenetic factors may lead to the discovery of new tumor suppressor genes. In lung cancer, the frequency of aberrant methylation of the p16INK4a gene increases during disease progression from basal cell hyperplasia to squamous metaplasia and carcinoma in situ lesions (16). ESCC is also thought to develop through a multi-stage process, from normal cells to hyperplasia, dysplasia, carcinoma in situ, and finally, carcinoma. In fact, hypermethylation of multiple genes has been detected in association with the development of ESCC (17). Discovery of genes with aberrant methylation in accordance with the multi-stage process of ESCC might be useful for the accurate diagnosis and prediction for the risk of disease progression.

Herein, we investigated the promoter methylation status of four genes and revealed aberrant methylation of the following three genes in ESCC cell lines: CTSL2, NMES1, and FABP5. NMES1 was identified due to its strikingly low or completely absent expression in clinical samples of ESCCs (18). Furthermore, the aberrant methylation of the NMES1 promoter region was present in a high proportion of cervical cancers, but not in normal samples (19). Therefore, we decided to investigate its function further. As for tissue distribution, the expression of NMES1 was observed in normal esophagus, 
TE1

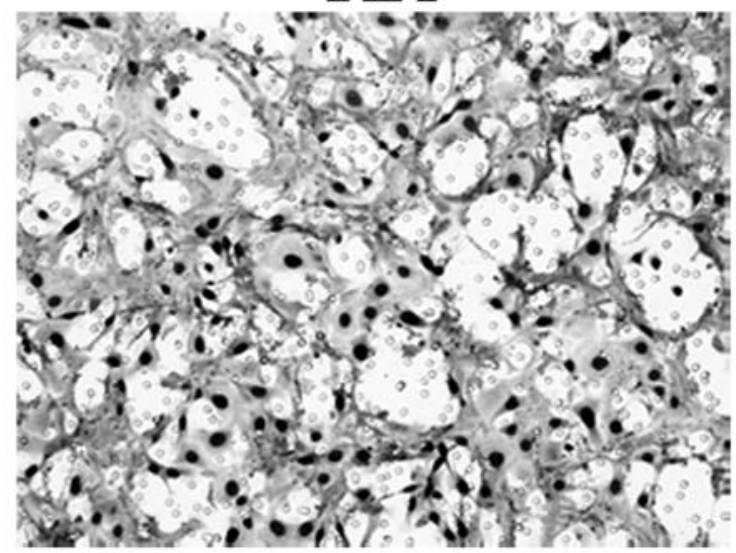

TE1-1

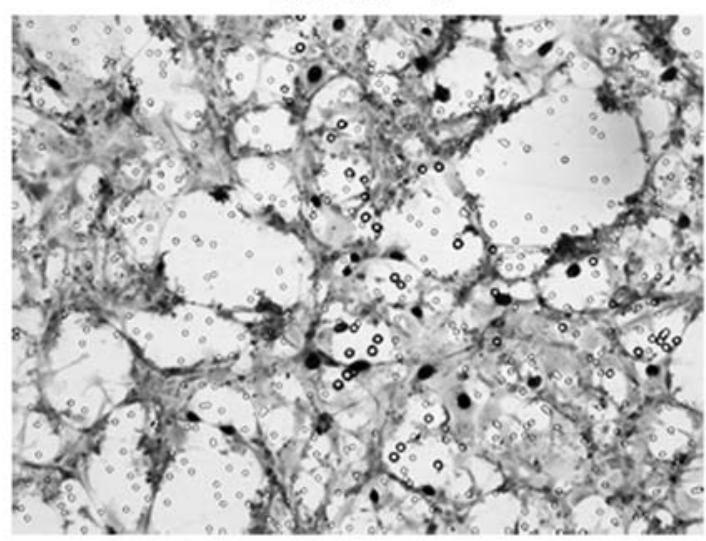

TE1-4

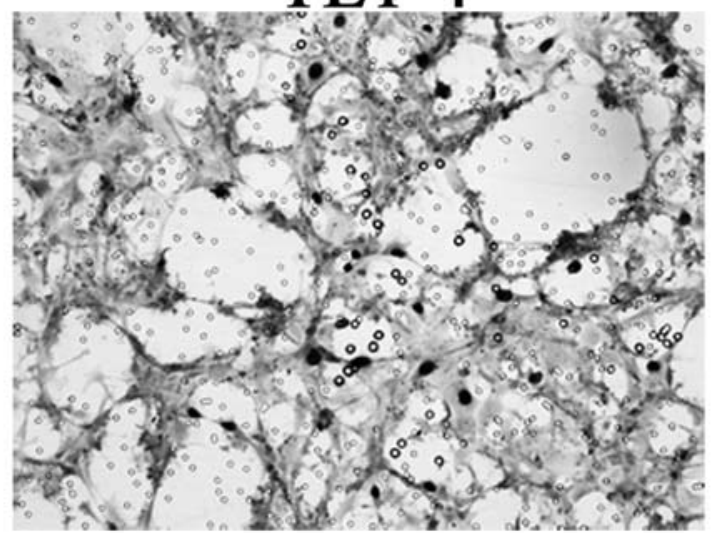

TE2

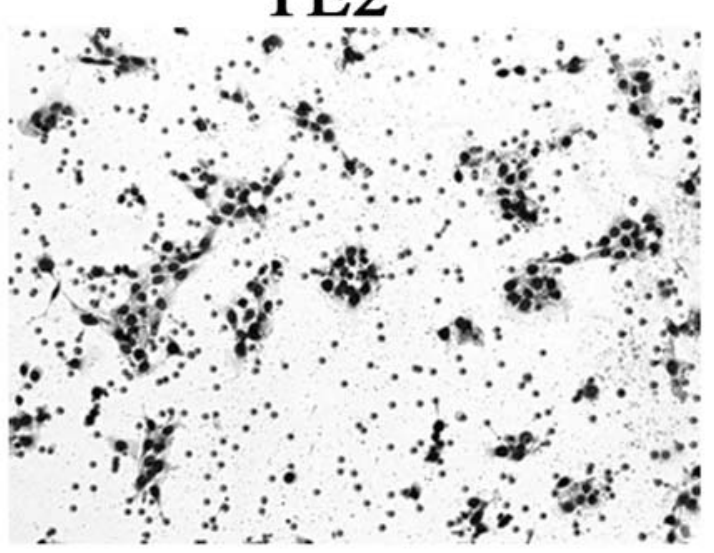

TE2-3

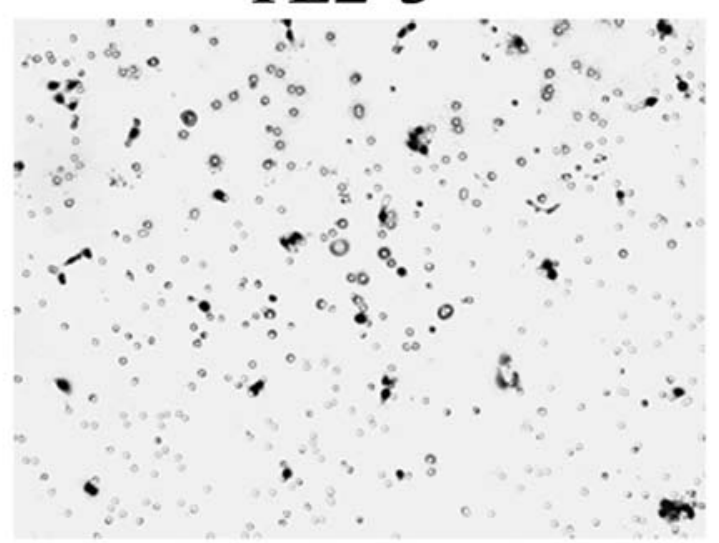

\section{TE2-5}

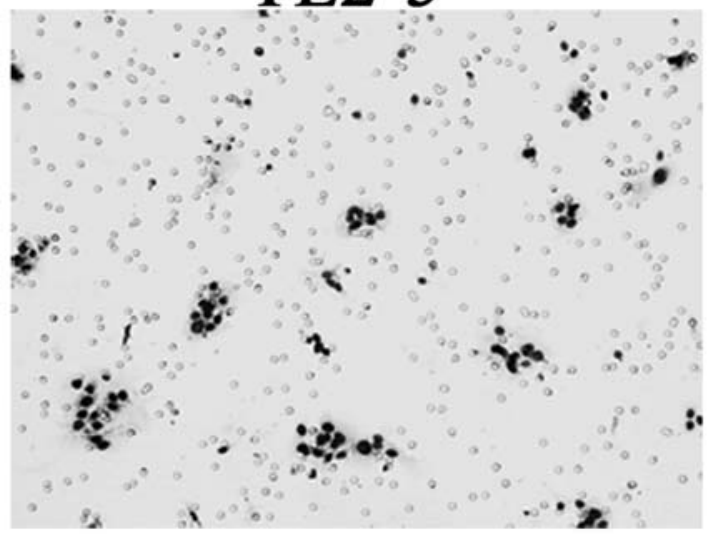

B

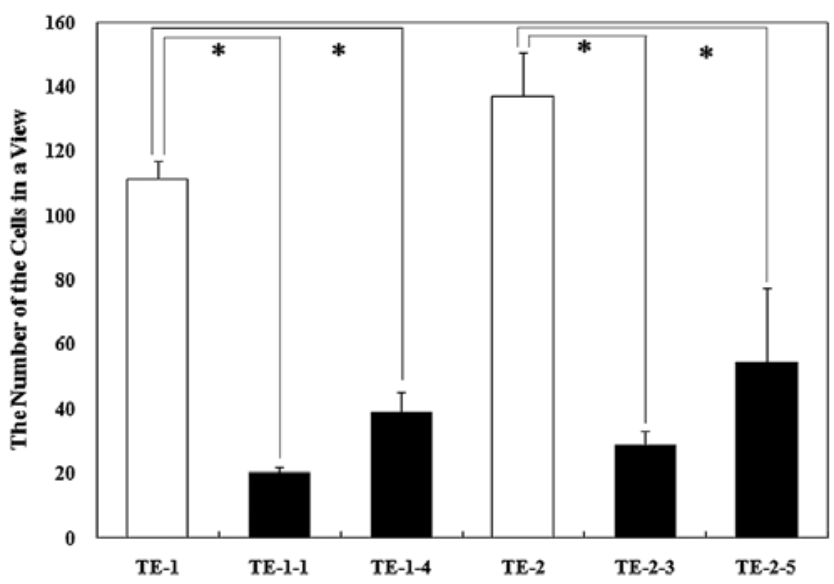

Figure 4. Effect of overexpression of normal mucosa of esophagus specific 1 (NMES1) on cell migration. We used the clones which expressed NMES1 stably, namely two sublines (clone TE1-1 and TE1-4) from TE-1, and two (clone TE2-3 and TE2-5) from TE-2. Boyden chamber assay was done to compare the migratory capabilities of NMES1 overexpressed cells with the parental ones. (A) The cells with overexpression of NMES1 (TE-1-1, TE1-4, TE2-3, and TE2-5) showed a decrease in the number of cells migrating compared with parental cells (TE-1 and TE-2). (B) The number of cells in a field of view were counted. There was a significant difference between NMES1 overexpressed cells and parental cells ( $\mathrm{p}<0.01$, Mann-Whitney U test). ${ }^{*} \mathrm{p}<0.01$. Error bars indicate S.D. 
stomach, small intestine, colon and placenta, with the NMES1 protein being located mainly in the nucleus, as determined by immunohistochemical analysis. In addition, NMES1 has been identified as a potential candidate for modifiers of susceptibility to skin tumor promotion by phorbol ester (20). In this study, using the Boyden chamber assay, we clarified that overexpression of NMES1 in ESCC cell lines could prohibit cell motility. We also performed the wound healing assay in TE-1, TE1-1, and TE1-4 according to a previous report (21). The speed of wound healing in TE-1 was faster than seen in TE1-1 and TE 1-4 (data not shown). Taken together, we speculated that NMES1 was related to cell motility of ESCC, and that maintenance of the expression of NMES1 in normal esophagus was important for the inhibition of the development of ESCC. To clarify this, further investigation using clinical samples is needed.

CTSL2 is a member of the papain family of cysteine proteinases and is highly expressed in colorectal and breast carcinomas, but not in normal colon, mammary gland, or peritumoral tissues. In addition, high expression levels of CTSL2 have been reported in ESCC; however, only a small number of samples were used in this study (22). FABP5 is a member of the fatty acid binding protein family that binds long-chain fatty acids (23). Overexpression of FABP5 has been observed in prostate cancer (24). FABP5 is a major target of c-Myc and has been shown to be rapidly up-regulated upon induction of EpCAM, which was itself up-regulated in squamous cell carcinoma of the head and neck $(25,26)$. Additionally, FABP5 levels have been shown to correlate with the amount of EpCAM in vivo in primary head and neck squamous cell carcinomas (25). Thus, it follows that FABP5 levels may be up-regulated in squamous cell carcinomas as well. In our study, the expressions of CTSL2 and FABP5 in cancer cell lines were down-regulated and restored by DAC treatment, which conflicted with the reports using clinical samples. Thus, the roles of CTSL2 and FABP5 in ESCC will need to be investigated further.

Transcript levels of GADD45A are increased following stressful growth arrest conditions and treatment with DNAdamaging agents. Wang et al reported that four CpGs, located $\sim 700$ bp upstream of the transcriptional start site, were methylated in the majority of breast cancer cell lines and clinical samples. Furthermore, this study showed that DAC induced the expression of GADD45A in breast cancer cell lines (27). In our study, the promoter region of $G A D D 45 A$ was slightly methylated, but almost identical to the methylated regions revealed by Wang et al. The methylation of this region might be important for the carcinogenesis of ESCC as well as breast cancer.

By DNA microarray-based transcriptomic analysis after DAC treatment, Yamashita et al have clarified previously the epigenetically silenced tumor suppressor genes in ESCC cell lines (28). However, the candidate genes silenced epigenetically in their study did not coincide with the ones silenced in ours. We speculate that the difference might be caused by using different microarrays or by the difference in the way candidate genes were selected from the microarray data.

In conclusion, we determined the methylation status, within the respective promoter regions, of CTSL2, NMES1, and
FABP5 in ESCC cell lines. Our results revealed that overexpression of NMES1 inhibits cell motility in ESCC cell lines. NMES1 might play an important role in metastasis of cancer cells and serve as a potential biomarker for ESCC.

\section{References}

1. Antequera $\mathrm{F}$ and Bird $\mathrm{A}$ : Number of $\mathrm{CpG}$ islands and genes in human and mouse. Proc Natl Acad Sci USA 90: 11995-11999, 1993.

2. Jones PA and Laird PW: Cancer epigenetics comes of age. Nat Genet 21: 163-167, 1999.

3. Baylin SB: Reversal of gene silencing as a therapeutic target for cancer - roles for DNA methylation and its interdigitation with chromatin. Novartis Found Symp 259: 226-237, 2004.

4. Xing EP, Nie Y, Song Y, et al: Mechanisms of inactivation of p14ARF, p15INK4b, and p16INK4a genes in human esophageal squamous cell carcinoma. Clin Cancer Res 5: 2704-2713, 1999.

5. Nie Y, Yang G, Song Y, et al: DNA hypermethylation is a mechanism for loss of expression of the HLA class I genes in human esophageal squamous cell carcinomas. Carcinogenesis 22: $1615-1623,2001$

6. Si HX, Tsao SW, Lam KY, et al: E-cadherin expression is commonly downregulated by $\mathrm{CpG}$ island hypermethylation in esophageal carcinoma cells. Cancer Lett 173: 71-78, 2001.

7. Yamaguchi S, Kato H, Miyazaki T, et al: RASSF1A gene promoter methylation in esophageal cancer specimens. Dis Esophagus 18: 253-256, 2005.

8. Suzuki H, Gabrielson E, Chen W, et al: A genomic screen for genes upregulated by demethylation and histone deacetylase inhibition in human colorectal cancer. Nat Genet 31: 141-149, 2002.

9. Issa JP, Ahuja N, Toyota M, Bronner MP and Brentnall TA: Accelerated age-related $\mathrm{CpG}$ island methylation in ulcerative colitis. Cancer Res 61: 3573-3577, 2001.

10. Fukai K, Yokosuka O, Chiba T, et al: Hepatocyte growth factor activator inhibitor 2/placental bikunin (HAI-2/PB) gene is frequently hypermethylated in human hepatocellular carcinoma. Cancer Res 63: 8674-8679, 2003.

11. Nishihira T, Hashimoto Y, Katayama M, Mori S and Kuroki T: Molecular and cellular features of esophageal cancer cells. J Cancer Res Clin Oncol 119: 441-449, 1993.

12. Arai M, Yokosuka O, Chiba T, et al: Gene expression profiling reveals the mechanism and pathophysiology of mouse liver regeneration. J Biol Chem 278: 29813-29818, 2003.

13. Takai D and Jones PA: Comprehensive analysis of CpG islands in human chromosomes 21 and 22. Proc Natl Acad Sci USA 99: 3740-3745, 2002.

14. Takai D and Jones PA: The CpG island searcher, a new WWW resource. In Silico Biol 3: 235-240, 2003.

15. Murphy RM, Watt KK, Cameron-Smith D, Gibbons CJ and Snow RJ: Effects of creatine supplementation on housekeeping genes in human skeletal muscle using real-time RT-PCR. Physiol Genomics 12: 163-174, 2003.

16. Belinsky SA, Nikula KJ, Palmisano WA, et al: Aberrant methylation of p16(INK4a) is an early event in lung cancer and a potential biomarker for early diagnosis. Proc Natl Acad Sci USA 95: 11891-11896, 1998

17. Nie Y, Liao J, Zhao X, et al: Detection of multiple gene hypermethylation in the development of esophageal squamous cell carcinoma. Carcinogenesis 23: 1713-1720, 2002.

18. Zhou J, Wang H, Lu A, et al: A novel gene, NMES1, downregulated in human esophageal squamous cell carcinoma. Int J Cancer 101: 311-316, 2002.

19. Sova P, Feng Q, Geiss G, et al: Discovery of novel methylation biomarkers in cervical carcinoma by global demethylation and microarray analysis. Cancer Epidemiol Biomarkers Prev 15: 114-123, 2006.

20. Riggs PK, Angel JM, Abel EL and Digiovanni J: Differential gene expression in epidermis of mice sensitive and resistant to phorbol ester skin tumor promotion. Mol Carcinog 44: 122-136, 2005.

21. Goulimari P, Kitzing TM, Knieling H, et al: Galpha12/13 is essential for directed cell migration and localized Rho-Dial function. J Biol Chem 280: 42242-42251, 2005.

22. Nawata S, Nakamura K, Hirakawa H, et al: Electrophoretic analysis of the cleaved form of serpin, squamous cell carcinoma antigen-1 in normal and malignant squamous epithelial tissues. Electrophoresis 24: 2277-2282, 2003. 
23. Madsen P, Rasmussen HH, Leffers H, Honore B and Celis JE: Molecular cloning and expression of a novel keratinocyte protein (psoriasis-associated fatty acid-binding protein [PA-FABP]) that is highly up-regulated in psoriatic skin and that shares similarity to fatty acid-binding proteins. J Invest Dermatol 99: 299-305, 1992.

24. Vanaja DK, Ballman KV, Morlan BW, et al: PDLIM4 repression by hypermethylation as a potential biomarker for prostate cancer. Clin Cancer Res 12: 1128-1136, 2006.

25. Munz M, Zeidler R and Gires O: The tumour-associated antigen EpCAM upregulates the fatty acid binding protein E-FABP. Cancer Lett 225: 151-157, 2005.
26. Andratschke M, Hagedorn H, Luebbers CW, et al: Limited suitability of EpCAM for molecular staging of tumor borders in head and neck cancer. Anticancer Res 26: 153-158, 2006.

27. Wang W, Huper G, Guo Y, Murphy SK, Olson JA Jr and Marks JR: Analysis of methylation-sensitive transcriptome identifies GADD45a as a frequently methylated gene in breast cancer. Oncogene 24: 2705-2714, 2005.

28. Yamashita K, Upadhyay S, Osada M, et al: Pharmacologic unmasking of epigenetically silenced tumor suppressor genes in esophageal squamous cell carcinoma. Cancer Cell 2: 485-495, 2002. 